\title{
The effect of starvation on the susceptibility of teneral and non-teneral tsetse flies to trypanosome infection
}

\author{
- $\quad$ C. Kubi ${ }^{1}$, \\ - J. Van Den Abbeele ${ }^{2}$, \\ - $\quad$ R. De Deken ${ }^{1}$, \\ - T. Marcotty ${ }^{1}$, \\ - $\quad$ P. Dorny ${ }^{1,3}$ \\ - P. Van Den Bossche $e^{1,4}$ \\ ${ }^{1}$ Department of Animal Health, \\ ${ }^{2}$ Department of Parasitology, Institute of Tropical Medicine, Antwerp, \\ ${ }^{3}$ Department of Virology, Parasitology and Immunology, Ghent University, Merelbeke, \\ Belgium and \\ ${ }^{4}$ Department of Veterinary Tropical Diseases, Faculty of Veterinary Sciences, University \\ of Pretoria, Pretoria, South Africa
}

[Tables at the bottom of the document]

\section{Abstract}

Transmission of vector-borne diseases depends largely on the ability of the insect vector to become infected with the parasite. In tsetse flies, newly emerged or teneral flies are considered the most likely to develop a mature, infective trypanosome infection. This was confirmed during experimental infections where laboratory-reared Glossina morsitans morsitans Westwood (Diptera: Glossinidae) were infected with Trypanosoma congolense or T. brucei brucei. The ability of mature adult tsetse flies to become infected with trypanosomes was significantly lower than that of newly emerged flies for both parasites. However, the nutritional status of the tsetse at the time of the infective bloodmeal affected its ability to acquire either a T. congolense or T. b. brucei infection. Indeed, an extreme period of starvation (3-4 days for teneral flies, 7 days for adult flies) lowers the developmental barrier for a trypanosome infection, especially at the midgut level of the tsetse fly. Adult G. m. morsitans became at least as susceptible as newly emerged flies to infection with T. congolense. Moreover, the susceptibility of adult flies, starved for 7 
days, to an infection with T. b. brucei was also significantly increased, but only at the level of maturation of an established midgut infection to a salivary gland infection. The outcome of these experimental infections clearly suggests that, under natural conditions, nutritional stress in adult tsetse flies could contribute substantially to the epidemiology of tsetse-transmitted trypanosomiasis.

\section{Introduction}

Tsetse-transmitted trypanosomiasis is a vector-borne disease that poses a serious threat to human and animal health in large parts of sub-Saharan Africa. Tsetse flies occur on about 10 million square $\mathrm{km}$ of the African continent. The epidemiology of the disease in humans and animals depends, among other factors, on the proportion of infected flies in a tsetse population. A wide range of intrinsic and extrinsic factors are proposed to determine the tsetse vector competence (reviewed by Leak, 1998). More recently, several studies have suggested that specific immune responses of the tsetse fly against the trypanosome parasite possibly interfere with the establishment and maturation of the trypanosome in the tsetse (reviewed by Aksoy et al., 2003). In most trypanosomiasis transmission models, the age-specific susceptibility of tsetse to a trypanosome infection is taken as an important parameter. Tsetse flies are believed to be most susceptible at their first bloodmeal (Buxton, 1955; Wijers, 1958; Distelmans et al., 1982; Welburn \& Maudlin, 1992) and subsequent trypanosome-infected bloodmeals contribute little to the overall infection rate of the tsetse population. However, field observations on the prevalence of trypanosome infections in various age categories of tsetse show a significant increase in the proportions of infected flies by age well above the increase that would be expected when taking into account the differences in susceptibility between teneral and non-teneral flies (Woolhouse \& Hargrove, 1998). These observations clearly indicate that a substantial proportion of the adult tsetse population still has the ability to pick up a trypanosome infection that matures into the mammalian infective stage. A few laboratory studies have already suggested that adult tsetse flies can acquire a mature Trypanosoma brucei brucei infection and that, in some cases, starvation increases their susceptibility to such an infection (Gingrich et al., 1982; Gooding, 1988; Welburn et al., 1989). To further clarify the potential role of the adult tsetse population in the transmission dynamics of African trypanosomiasis, the age-related ability of tsetse flies to develop mature T. congolense and T. b. brucei infections was reconsidered. Moreover, this study attempted to clarify the effect of starvation on the modulation of this vectoral ability. 


\section{Materials and methods}

\section{Tsetse flies and trypanosome strains}

Male Glossina morsitans morsitans Westwood from the colony maintained at the Institute of Tropical Medicine (Antwerp, Belgium) were used in all experiments. The origin of the colony and its maintenance have been described by Elsen et al. (1993).

The trypanosome strains used in the experiments were a $T . b$. brucei strain derived from the stock EATRO 1125 AnTAR1 (Le Ray et al., 1977) and T. congolense IL 1180, a strain originating from Serengeti in Tanzania (Geigy \& Kauffman, 1973).

\section{Experimental design}

Male G. m. morsitans of various ages (teneral and non-teneral flies) were given an infective bloodmeal with either T. congolense or T. b. brucei. In this context we define teneral flies as newly emerged, soft-bodied flies that had not received a bloodmeal prior to the infective feed, whereas non-tenerals had received at least one bloodmeal prior to the infective bloodmeal. For the single infective feed, a batch of 40 flies was fed on an anaesthetized mouse showing a blood parasitaemia of T. congolense or T. b. brucei of approximately $10^{8.4}$ trypanosomes $/ \mathrm{mL}$. Only fully engorged flies were retained and were maintained on clean rabbits. To avoid re-infection of the flies, these rabbits were replaced at weekly intervals. For each experimental series, three to five batches of flies were infected separately.

Surviving flies were dissected 20 days (for T. congolense-infected flies) and 30 days (for T. b. brucei-infected flies) after the infective feed, according to the method described by Lloyd \& Johnson (1924). The midgut and mouthparts or salivary glands were examined for the presence of trypanosomes.

In the first experiment, the age-specific susceptibility to infection of teneral and nonteneral male G. m. morsitans was determined. For this purpose, 0-2-day-old teneral flies and 4-20-day-old non-teneral G. m. morsitans were infected and their infection status determined. In a subsequent experiment, the effect of starvation on susceptibility to infection was assessed by infecting teneral flies and 20-day-old adult flies after starving them for 1-4 days and for 2, 3, 5 or 7 days, respectively.

The prevalence of procyclic midgut infections was calculated as the proportion of dissected flies that had a trypanosome infection in the midgut. The prevalence of mature

or metacyclic infections was calculated as the proportion of dissected flies infected in the 
proboscis (for infections with T. congolense) or salivary glands (for infections with T. $b$. brucei). The maturation of an infection was calculated as the proportion of midgut infections that developed into a mature infection. In the first experiment, the proportion of midgut and metacyclic infections and maturation of infections in the teneral and nonteneral flies were compared using chi-square tests. Data obtained from the second experiment determining the effect of starvation (categorical explanatory variable) were analysed using logistic regression with stata Release 8.0 (Stata Corp., College Station, TX, U.S.A.). Three logistic regressions were calculated for each trypanosome species. The response variables were the proportion of flies infected in the midgut, the proportion of metacyclic infections and the maturation of infections. When a logistic regression failed because of proportions equal to 0 or 1 , the exact method was used to calculate a confidence interval. Animal ethics approval for the experimental infections was obtained from the Animal Ethical Commission of the Institute of Tropical Medicine, Antwerp, Belgium (ref. DG001-PD-M-TT).

\section{Results}

\section{Age-specific susceptibility to infection}

The uptake of a single bloodmeal prior to the infective bloodmeal significantly reduced tsetse fly ability to establish a procyclic midgut infection with T. congolense or T. $b$. brucei $(P<0.001)$ (Table 1, Fig. 1A, B). Moreover, the maturation of T. brucei midgut infection was also significantly reduced in non-teneral flies. Despite this, a substantial proportion of the flies infected in the non-teneral stage were still able to acquire a mature infection (i.e. $11 \%$ and $5 \%$ of flies given an infective bloodmeal with T. congolense and T. b. brucei, respectively).

\section{Starvation and susceptibility to infection}

In non-teneral flies, an extreme starvation period of 7 days resulted in a significant increase in the proportion of flies that developed a mature infection with $T$. congolense or T. b. brucei (Tables 2 and 3). For T. congolense, this was exclusively due to the increased ability of the flies to develop an established procyclic midgut infection. By contrast, for a T. b. brucei infection, the starvation of adult flies did not have any effect on the establishment of a midgut infection but resulted in an increased maturation rate (i.e. a higher proportion of midgut infections giving rise to mature, metacyclic infections in the salivary glands). 
The effect of starvation on the susceptibility of tsetse flies was also clearly demonstrated in teneral flies. Here, starvation for 3-4 days prior to the infective bloodmeal significantly increased the proportion of flies that established a T. congolense or T. $b$. brucei midgut infection (Tables 2 and 3).

\section{Discussion}

These data confirm that the age of a tsetse fly at the time of the infective bloodmeal drastically affects the vectoral ability of the fly for T. congolense as well as T. b. brucei, as has been observed earlier (Distelmans et al., 1982; Welburn \& Maudlin, 1992). Contrary to the observations made by Wijers (1958), who infected G. palpalis with T. $b$. gambiense, a decrease in the prevalence of infections in flies infected at the 2-day-old teneral stage was not observed here. The vectoral ability is highest in newly emerged flies and decreases substantially when a tsetse fly has taken a single bloodmeal before the infective one. Nevertheless, the ability of adult flies to acquire a mature infection remains relatively high, especially for $T$. congolense. This is in line with field observations made by Woolhouse et al. $(1993,1994)$ on the age-specific infection rates of G. pallidipes with T. congolense. Contrary to findings by Welburn \& Maudlin (1992), maturation of $T$. congolense midgut infections in non-teneral flies did not differ significantly from maturation in teneral flies.

The nutritional status of the tsetse fly at the time of the infective bloodmeal affects its vectoral ability for T. congolense as well as T. b. brucei. Indeed, an extreme period of starvation (3-4 days for teneral flies, 7 days for adult flies) lowers the developmental barrier for a trypanosome infection, especially at the midgut level of the tsetse fly. This suggests that the physiological factors that constitute this barrier are suppressed when the fly is under nutritional stress. Starvation possibly reduces the immunological responses of the fly to invading microorganisms such as trypanosomes. Indeed, it is assumed that components of the insect immune system are involved in the natural refractoriness observed in the tsetse fly (reviewed by Aksoy et al., 2003). Recent studies have demonstrated an induced expression and synthesis of several antimicrobial peptides when flies are offered a trypanosome-infected bloodmeal (Hao et al., 2001; Boulanger et al., 2002). The fat body constitutes the tsetse energy reserve but also functions as a key centre of metabolism and biochemistry control for the fly innate immune response. The energy cost to the insect of using this immune defence machinery is considerable (Schmid-Hempel, 2005). Hence, when a tsetse fly is exposed to nutritional stress up to a level that causes serious depletion of its energy reserves, it is plausible that the tsetse 
immune functions are downregulated, leading to a less hostile midgut environment for ingested trypanosomes. This may explain the increase in midgut infection rates observed for trypanosome infections with $T$. congolense in both teneral flies and adult flies starved for 7 days. Although this was not observed in the infection of adult flies starved for 7 days with $T$. $b$. brucei, the significantly higher trypanosome maturation rate observed in these flies may also be explained by this less hostile midgut environment, as the growth of the procyclic trypanosome population in the tsetse midgut will be less hindered, increasing the probability that the infection will progress to the tsetse salivary glands (Dale et al., 1995). The relationship between nutritional stress and immune response may also explain why the effect of starvation on the tsetse's susceptibility to infection is only observed after the flies have been starved for several days (i.e. after the lipid reserve has been depleted sufficiently to affect immune responses). Moreover, the reverse phenomenon could explain why feeding teneral flies results in a higher developmental barrier for trypanosomes at the tsetse midgut level. Indeed, teneral flies are physiologically immature, have limited energy reserves and need a bloodmeal (or several) to make all physiological systems fully operational, including immune response.

In conclusion, more experimental evidence is clearly needed to clarify the role of the immune system in trypanosome development and the impact of nutritional stress on this defence mechanism. Although the observations presented here are based entirely on laboratory experiments, the implications of these findings on the understanding of the epidemiology of animal and human trypanosomiasis may be considerable. First, the proportion of infected flies in a population may not be determined only by the proportion of teneral flies that take a bloodmeal on an infected host, but also by environmental factors, such as high ambient temperature, that may significantly reduce the tsetse's energy reserves. It is thus plausible that, during certain periods of the year, the overall infection rate of a tsetse population is determined to a large extent by a substantial number of non-teneral flies that acquire a mature infection. Furthermore, these findings may have repercussions for the distribution and prevalence of infections with trypanosome species that are difficult to transmit, for example, T. b. rhodesiense (Welburn et al., 1995). It could be hypothesized that the focal nature of the distribution of 'Rhodesiense' human sleeping sickness is due to local conditions that enhance the susceptibility of non-teneral flies to infection and thus substantially increase the proportion of flies in that particular population that can become infected and transmit the parasite. 


\section{References}

Aksoy, S., Gibson, W.C. \& Lehane, M.J. (2003) Interactions between tsetse and trypanosomes with implications for the control of trypanosomiasis. Advances in Parasitology, 53, 1-83.

-

Boulanger, N., Brun, R., Ehret-Sabatier, L., Kunz, C. \& Bulet, P. (2002) Immunopeptides in the defence reactions of Glossina morsitans to bacterial and Trypanosoma brucei brucei infections. Insect Biochemistry and Molecular Biology, 32, 369-375.

Buxton, P.A. (1955) The natural history of tsetse flies. H.K. Lewis Ltd., .

-

Dale, C., Welburn, S.C., Maudlin, I. \& Milligan, P.J.M. (1995) The kinetics of maturation of trypanosome infections in tsetse. Parasitology, 111, 187-191.

Distelmans, W., D'Haeseleer, F., Kaufman, L. \& Rousseeuw, P. (1982) The susceptibility of Glossina palpalis palpalis at different ages to infection with Trypanosoma congolense. Annales de la Société Belge de Medicine Tropicale, 62, 41-47.

Elsen, P., Van Hees, J. \& De Lil, E. (1993) L'historique et les conditions d'élevage des lignées de glossines (Diptera Glossinidae) maintenues á l'Institut de Médicine Tropicale Prince Léopold d'Anvers. Journal of African Zoology, 107, 439-499.

Geigy, R. \& Kauffman, M. (1973) Sleeping sickness survey in the Serengeti area (Tanzania) 1971. I. Examination of large mammals for trypanosomes. Acta Tropica, 30, $12-23$.

Gingrich, J.B., Ward, R.A., Macken, L.M. \& Esser, K.M. (1982) African sleeping sickness: new evidence that mature tsetse flies (Glossina morsitans) can become potent vectors. Transactions of the Royal Society of Tropical Medicine and Hygiene, 76, 479481.

Gooding, R. (1988) Infection of post-teneral tsetse flies (Glossina morsitans morsitans and Glossina morsitans centralis) with Trypanosoma brucei brucei. Canadian Journal of Zoology, 66, 1289-1292.

Hao, Z., Kasumba, I., Lehane, M.J., Gibson, W.C., Kwon, J. \& Aksoy, S. (2001) Tsetse immune responses and trypanosome transmission: implications for the development of tsetse-based strategies to reduce trypanosomiasis. Proceedings of the National Academy of Sciences of the USA, 98, 12648-12653.

Le Ray, D., Barry, J.D., Easton, C. \& Vickerman, K. (1977) First tsetse fly transmission of the 'Antat' serodeme of Trypanosoma brucei. Annales de la Société Belge de Medicine Tropicale, 57, 369-381. 
Leak, S.G.A. (1998) Tsetse Biology and Ecology: their Role in the Epidemiology and Control of Trypanosomosis. CABI Publishing, .

Lloyd, L.L. \& Johnson, W.B. (1924) The trypanosome infections of tsetse flies in Northern Nigeria and a method of estimation. Bulletin of Entomological Research, 14, 225-227.

-

Schmid-Hempel, P. (2005) Evolutionary ecology of insect immune defences. Annual Review of Entomology, 50, 529-551.

Welburn, S.C. \& Maudlin, I. (1992) The nature of the teneral state in Glossina and its role in the acquisition of trypanosome infection in tsetse. Annals of Tropical Medicine and Parasitology, 86, 529-536.

Welburn, S.C., Maudlin, I. \& Ellis, D.S. (1989) Rate of trypanosome killing by lectins in midguts of different species and strains of Glossina. Medical and Veterinary Entomology, $3,77-82$.

Welburn, S.C., Maudlin, I. \& Milligan, P.J.M. (1995) Trypanozoon: infectivity to humans is linked to reduced transmissibility in tsetse. I. Comparison of human serumresistant and human serum-sensitive field isolates. Experimental Parasitology, 81, 404408.

Wijers, D.J.B. (1958) Factors that may influence the infection rate of Glossina palpalis with Trypanosoma gambiense. I. The age of the fly at the time of the infected feed. Annals of Tropical Medicine and Parasitology, 52, 385-390.

Woolhouse, M.E.J., Bealby, K., McNamara, J.J. \& Silutongwe, J. (1994) Trypanosome infections of the tsetse fly Glossina pallidipes in the Luangwa Valley, Zambia. International Journal of Parasitology, 24, 987-993.

-

Woolhouse, M.E.J. \& Hargrove, J.W. (1998) On the interpretation of age-prevalence curves for trypanosome infections of tsetse flies. Parasitology, 116, 149-156.

Woolhouse, M.E.J., Hargrove, J.W. \& McNamara, J.J. (1993) Epidemiology of trypanosome infections of the tsetse fly Glossina pallidipes in the Zambezi Valley. Parasitology, 106, 479-485. 


\section{Tables}

Table 1. Overall midgut infection and maturation rate of male G. m. morsitans infected with T. congolense or T. b. brucei in a teneral (no bloodmeals prior to infection) or nonteneral status (one or more bloodmeals prior to infection).

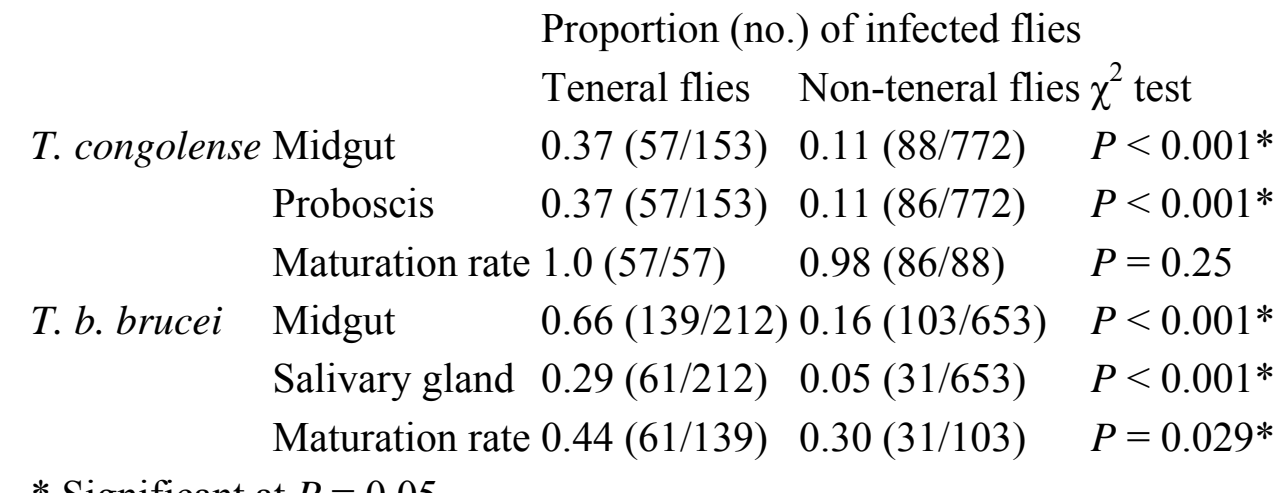

* Significant at $P=0.05$.

Table 2. Proportion of male G. m. morsitans infected with T. congolense after a period of starvation.

\begin{tabular}{|c|c|c|c|c|c|}
\hline \multirow[t]{2}{*}{ Flies } & \multirow{2}{*}{$\begin{array}{l}\text { Days of } \\
\text { starvation }\end{array}$} & \multirow{2}{*}{$\begin{array}{l}\text { Number of flies } \\
\text { dissected }\end{array}$} & \multicolumn{3}{|c|}{$\begin{array}{l}\text { Proportion of infected flies/maturation } \\
\text { with } 95 \% \text { CIs }\end{array}$} \\
\hline & & & Midgut & Proboscis & Maturation \\
\hline \multirow[t]{5}{*}{ Teneral flies } & 0 & 27 & $\begin{array}{l}0.22(0.10- \\
0.41)\end{array}$ & $\begin{array}{l}0.22(0.10- \\
0.41)\end{array}$ & $\begin{array}{l}1.00(0.61- \\
1.00)\end{array}$ \\
\hline & 1 & 75 & $\begin{array}{l}0.29(0.20- \\
0.40)\end{array}$ & $\begin{array}{l}0.29(0.20- \\
0.40)\end{array}$ & $\begin{array}{l}1.00(0.87- \\
1.00)\end{array}$ \\
\hline & 2 & 89 & $\begin{array}{l}0.22(0.15- \\
0.32)\end{array}$ & $\begin{array}{l}0.21(0.14- \\
0.31)\end{array}$ & $\begin{array}{l}0.95(0.72- \\
0.99)\end{array}$ \\
\hline & 3 & 71 & $\begin{array}{l}0.56(0.44- \\
0.67)\end{array}$ & $\begin{array}{l}0.56(0.44- \\
0.67)\end{array}$ & $\begin{array}{l}1.00(0.93- \\
1.00)\end{array}$ \\
\hline & 4 & 34 & $\begin{array}{l}0.56(0.39- \\
0.71)\end{array}$ & $\begin{array}{l}0.50(0.34- \\
0.66)\end{array}$ & $\begin{array}{l}0.89(0.66- \\
0.97)\end{array}$ \\
\hline \multirow[t]{3}{*}{$\begin{array}{l}20 \text {-day-old } \\
\text { adults }\end{array}$} & 2 & 75 & $\begin{array}{l}0.13(0.07- \\
0.23)\end{array}$ & $\begin{array}{l}0.09(0.04- \\
0.18)\end{array}$ & $\begin{array}{l}0.70(0.38- \\
0.90)\end{array}$ \\
\hline & 3 & 71 & $\begin{array}{l}0.10(0.05- \\
0.19)\end{array}$ & $\begin{array}{l}0.08(0.04- \\
0.18)\end{array}$ & $\begin{array}{l}0.86(0.42- \\
0.98)\end{array}$ \\
\hline & 5 & 103 & $0.17\left(0.10_{-}\right.$ & $0.17\left(0.10_{-}\right.$ & $1.00(0.84-$ \\
\hline
\end{tabular}



Flies $\quad \begin{array}{ll}\text { Days of } & \text { Number of flies }\end{array} \quad \begin{aligned} & \text { Proportion of infected flies/maturation } \\ & \text { with 95\% CIs }\end{aligned}$ starvation dissected Midgut Proboscis Maturation $0.25) \quad 0.25) \quad 1.00)$

$\begin{array}{lllll}7 & 77 & 0.49(0.38- & 0.48(0.37- & 0.97(0.83- \\ & 0.60) & 0.59) & 0.99)\end{array}$

$95 \% \mathrm{CI}=95 \%$ confidence interval.

Table 3. Proportion of male G. m. morsitans infected with T. b. brucei after a period of starvation.

\begin{tabular}{|c|c|c|c|c|c|}
\hline \multirow{2}{*}{ Flies } & \multirow{2}{*}{$\begin{array}{l}\text { Days of } \\
\text { starvation }\end{array}$} & \multirow{2}{*}{$\begin{array}{l}\text { Number of flies } \\
\text { dissected }\end{array}$} & \multicolumn{3}{|c|}{$\begin{array}{l}\text { Proportion of infected flies/maturation } \\
\text { with } 95 \% \text { CIs }\end{array}$} \\
\hline & & & Midgut & $\begin{array}{l}\text { Salivary } \\
\text { gland }\end{array}$ & Maturation \\
\hline \multirow[t]{5}{*}{ Teneral flies } & 0 & 69 & $\begin{array}{l}0.45(0.34- \\
0.57)\end{array}$ & $\begin{array}{l}0.22(0.14- \\
0.33)\end{array}$ & $\begin{array}{l}0.48(0.32- \\
0.65)\end{array}$ \\
\hline & 1 & 71 & $\begin{array}{l}0.42(0.31- \\
0.54)\end{array}$ & $\begin{array}{l}0.21(0.13- \\
0.32)\end{array}$ & $\begin{array}{l}0.50(0.33- \\
0.67)\end{array}$ \\
\hline & 2 & 68 & $\begin{array}{l}0.37(0.26- \\
0.49)\end{array}$ & $\begin{array}{l}0.19(0.11- \\
0.30)\end{array}$ & $\begin{array}{l}0.52(0.33- \\
0.70)\end{array}$ \\
\hline & 3 & 52 & $\begin{array}{l}0.65(0.52- \\
0.77)\end{array}$ & $\begin{array}{l}0.40(0.28- \\
0.54)\end{array}$ & $\begin{array}{l}0.62(0.45- \\
0.76)\end{array}$ \\
\hline & 4 & 44 & $\begin{array}{l}0.77(0.63- \\
0.87)\end{array}$ & $\begin{array}{l}0.41(0.28- \\
0.56)\end{array}$ & $\begin{array}{l}0.53(0.36- \\
0.68)\end{array}$ \\
\hline \multirow[t]{4}{*}{$\begin{array}{l}20 \text {-day-old } \\
\text { adults }\end{array}$} & 2 & 95 & $\begin{array}{l}0.21(0.22- \\
0.40)\end{array}$ & $\begin{array}{l}0.06(0.03- \\
0.13)\end{array}$ & $\begin{array}{l}0.21(0.10- \\
0.39)\end{array}$ \\
\hline & 3 & 96 & $\begin{array}{l}0.14(0.08- \\
0.22)\end{array}$ & $\begin{array}{l}0.06(0.03- \\
0.13)\end{array}$ & $\begin{array}{l}0.46(0.22- \\
0.72)\end{array}$ \\
\hline & 5 & 67 & $\begin{array}{l}0.22(0.14- \\
0.34)\end{array}$ & $\begin{array}{l}0.06(0.02- \\
0.15)\end{array}$ & $\begin{array}{l}0.27(0.10- \\
0.53)\end{array}$ \\
\hline & 7 & 64 & $\begin{array}{l}0.25(0.16- \\
0.37)\end{array}$ & $\begin{array}{l}0.16(0.09- \\
0.27)\end{array}$ & $\begin{array}{l}0.63(0.38- \\
0.82)\end{array}$ \\
\hline
\end{tabular}

$95 \% \mathrm{CI}=95 \%$ confidence interval. 